\title{
Spectral snow-reflectance models for grain-size and liquid- water fraction in melting snow for the solar-reflected spectrum
}

\author{
Robert O. Green, ${ }^{1,2}$ Jeff Dozier, ${ }^{2}$ Dar Roberts, ${ }^{2}$ Tom Painter ${ }^{2}$ \\ ${ }^{1}$ Jet Propulsion Laboratory, California Institute of Technology, 4800 Oak Grove Drive, Pasadena, CA 91109-8099, U.S.A. \\ ${ }^{2}$ Institute for Computational Earth System Science at the University of California, Santa Barbara, CA 93106, U.S.A.
}

\begin{abstract}
Two spectral snow-reflectance models that account for the effects of grain-size and liquid-water fraction are described and initial validation results presented. The models are based upon the spectral complex refractive index of liquid water and ice in the region from 400 to $2500 \mathrm{~nm}$. Mie scattering calculations are used to specify the essential optical properties of snow in the models. Two approaches are explored to model the effect of liquid water in the snow. The first accounts for the liquid water as separate spheres interspersed with ice spheres in the snow layer. The second accounts for the liquid water as coatings on ice grains in the snow layer. A discrete-ordinate radiative transfer code is used to model the spectral reflectance of the snow for the Mie-calculated optical properties. Both the interspersed- and coated-sphere models show that the snow-absorption feature at $1030 \mathrm{~nm}$ shifts to shorter wavelength as the liquid-water content increased. The expression of these shifts is different for the two models. A comparison of the models with a spectral measurement of frozen and melting snow shows better agreement with the coated-sphere model. A spectral fitting algorithm was developed and tested with the coated-sphere model to derive the grain-size and liquid-water fraction from snow spectral reflectance measurements. Consistent values of grain-size and liquid water were retrieved from the measured snow spectra. This research demonstrates the use of spectral models and spectral measurements to derive surface snow grain-size and liquid-water fraction. The results of this research may be extended to regional and greater scales using data acquired by airborne and spaceborne imaging spectrometers for contributions to energy balance and hydrological modeling.
\end{abstract}

\section{INTRODUGTION}

Two important properties of snow are surface grain-size and liquid-water fraction. The albedo of snow, which affects the energy balance, is a function of grain-size. The distribution of liquid water released from snow, which impacts the hydrology, is a function of the amount of melting. Measuring the distribution of grain-size and snowmelt from an Earth remote-sensing perspective would contribute to current and future Earth energy-balance and hydrological modeling and prediction. Previous snow-modeling and measurement work has shown that snow spectral reflectance is a strong function of grain-size and may be modeled from the optical properties of ice (Bohren and Barkstrom, 1974; Wiscombe and Warren, 1980; Warren 1982; Dozier, 1989; Nolin and Dozier 1993; Painter and others, 1998; Nolin and Dozier 2000). The research presented here describes, evaluates and tests two new snow-reflectance models that account for both grainsize and liquid-water fraction.

\section{SNOW MODELS}

The snow models developed here are based upon the inherent optical properties of pure ice and liquid water. These properties are reported in the form of the complex refractive index of ice and liquid water (Warren, 1982; Kou and others, 1993) shown in Figure 1. For both ice and liquid water the real component of the refractive index varies slightly across the spectral range $400-2500 \mathrm{~nm}$. In contrast, the imaginary component varies by seven orders of magnitude and contains several local minima. Of interest for this research is the displacement of the minima at $1030 \mathrm{~nm}$ for ice to $980 \mathrm{~nm}$ for liquid water. This displacement provides a basis for a difference in the spectrum of frozen vs melting snow that contains liquid water.

To develop the snow models, the refractive indices were used to calculate the required extinction, scattering and asymmetry properties of snow grains modeled as spheres with Mie computations (Wiscombe, 1980). The Mie-calculated

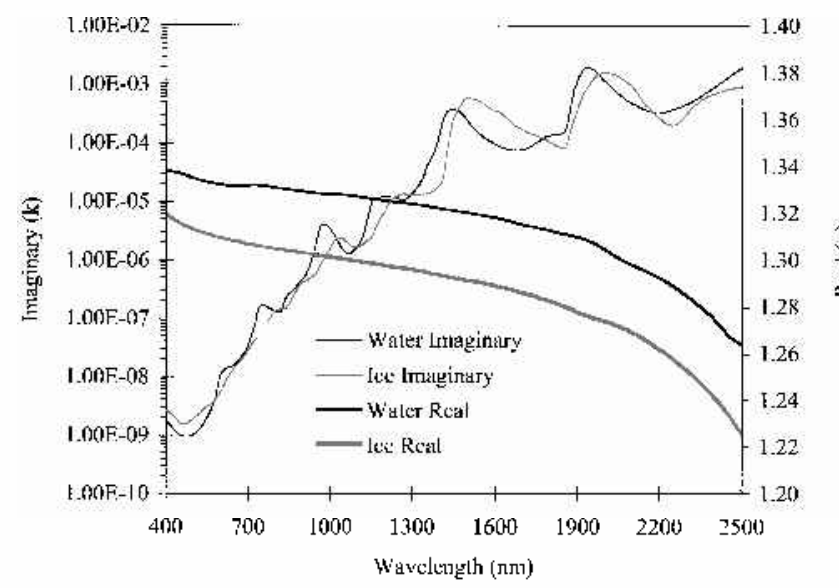

Fig. 1. Real and imaginary component of the refractive index of ice and liquid water. 


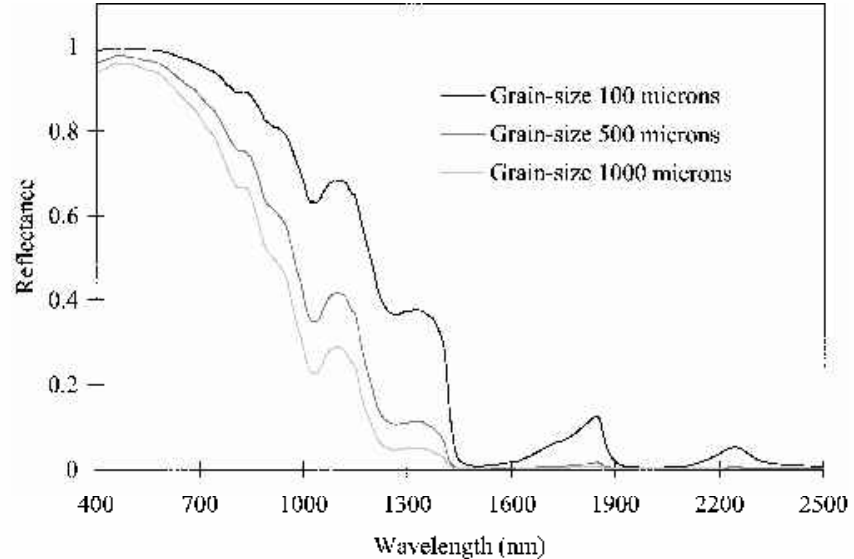

Fig. 2. Modeled frozen-snow reflectance for grain-sizes of 100, 500 and $1000 \mu \mathrm{m}$.

optical properties were then used to constrain a discreteordinate radiative transfer code (DISORT) (Stamnes and others, 1988) and model the spectral reflectance of snow. Figure 2 shows the calculated spectral reflectance of frozen snow for grain-sizes of 100,500 and $1000 \mu \mathrm{m}$. The modeled spectra show the expected high reflectance from 400 to $800 \mathrm{~nm}$, the strongly grain-size-dependent reflectance from 800 to $1400 \mathrm{~nm}$, and the comparatively low reflectance from 1400 to $2500 \mathrm{~nm}$. The local snow-reflectance minimum at $1030 \mathrm{~nm}$ is well expressed at all grain-sizes.
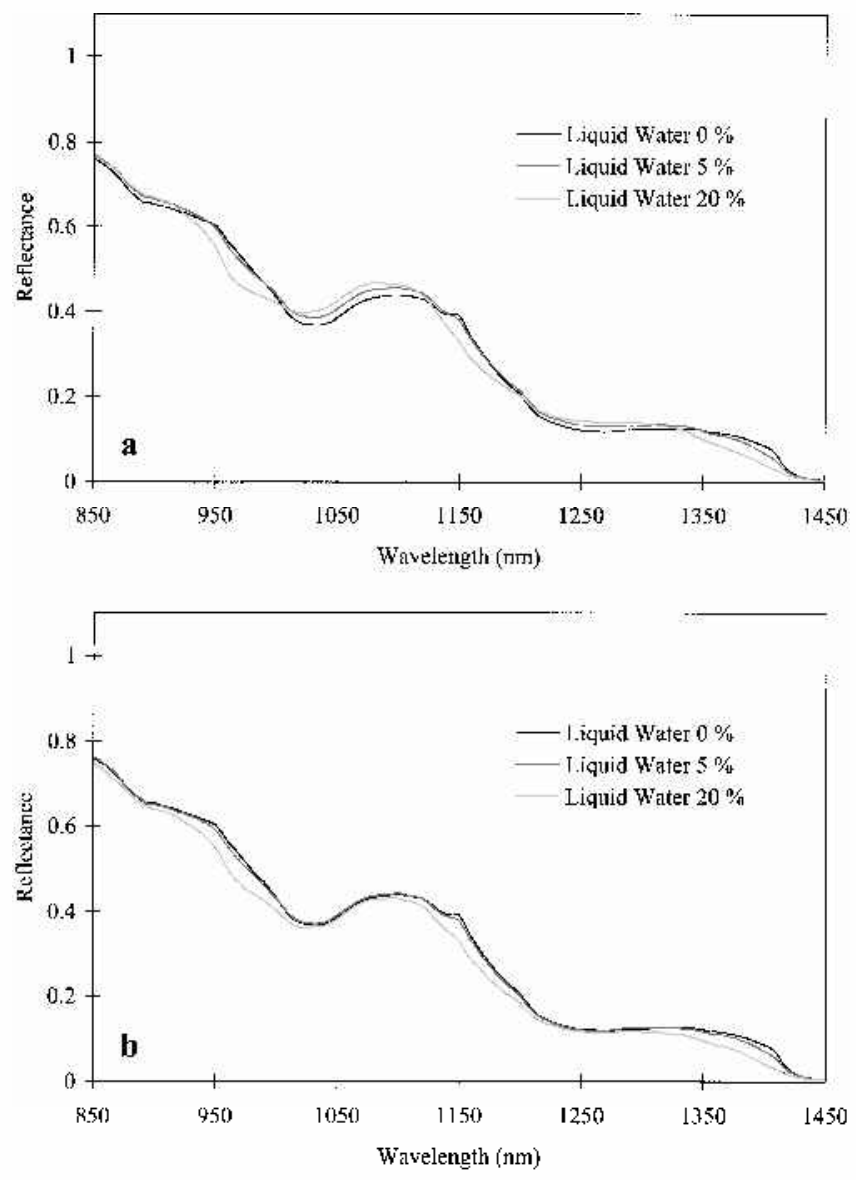

Fig. 3. Modeled melting-snow reflectance for a grain-size of $500 \mu \mathrm{m}$ with liquid-water mass fraction of $0 \%, 5 \%$ and $25 \%$. (a) The snow is modeled as interspersed spheres of ice and liquid water. (b) The liquid water is modeled as coated spheres with ice cores and liquid-water shells.

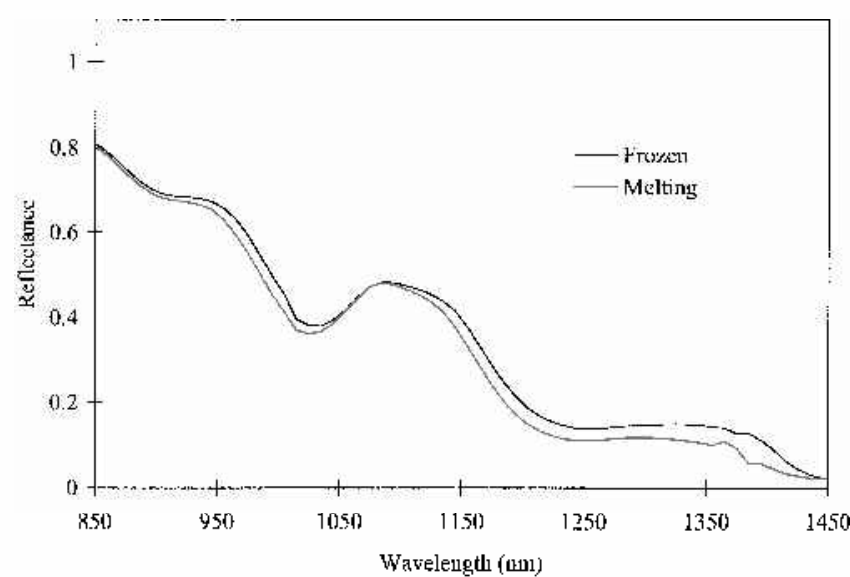

Fig. 4. Spectra from a single snow sample in a frozen and melting state.

To include the effect of liquid water, two approaches were explored. The first approach models the effect of liquid water as interspersed ice and water spheres. This approximates the case where water is filling some portion of the interstitial spaces of the snow. Mie calculations were performed for ice grains and liquid-water spheres. These calculated parameters were combined in area-weighted averages (Warren and Wiscombe, 1980) to provide the extinction, scattering and asymmetry properties of the snow. DISORT was used to model the spectral reflectance of frozen and melting snow shown in Figure 3a. As the fraction of liquid water increases, the $1030 \mathrm{~nm}$ snow-absorption feature in the modeled spectra shifts to shorter wavelengths. The second approach to model the effect of liquid water uses a layered-sphere model. Layered-sphere Mie calculations (Toon and Ackerman, 1981) were used to calculate the extinction, scattering and asymmetry properties of snow grains with liquid-water shells. These Mie parameters were then used to constrain DISORT to model the reflectance of snow. The DISORT model is directional, allowing specification of illumination and observation angles. Figure $3 \mathrm{~b}$ shows the modeled spectral reflectance for the layered-sphere approach. As with the interspersed-sphere model, the $1030 \mathrm{~nm}$ absorption feature shifts to shorter wavelengths with increasing liquid-water content. As expected based on the different melting-snow model assumptions, the form of the spectral shift is different between the interspersedand layered-sphere models.

\section{INITIAL VALIDATION}

As an initial test of the models, spectral reflectance measurements of a frozen and melting snow sample were acquired. A 30 by 30 by $30 \mathrm{~cm}$ block of snow with an estimated grain-size of $500 \mu \mathrm{m}$ was collected and placed in a freezer at $-20^{\circ} \mathrm{C}$. To measure the snow a portable field spectrometer (ASDFR, ASD Inc., Boulder, CO 80301) with a wavelength range of $350-2500 \mathrm{~nm}$ with nominally $10 \mathrm{~nm}$ spectral resolution was used. A spectralon reference target (Labsphere Inc., North Suton, NH 03620) was used as a reflectance calibration standard. The spectrometer was configured to measure spectra every $30 \mathrm{~s}$. The frozen-snow sample was removed from the freezer and placed in the field of view of the spectrometer under clear-sky solar illumination. The solar zenith angle was $36^{\circ}$ and air temperature was $23^{\circ} \mathrm{C}$. A time series of measurements was acquired as the snow warmed to $0^{\circ} \mathrm{C}$ and began to 

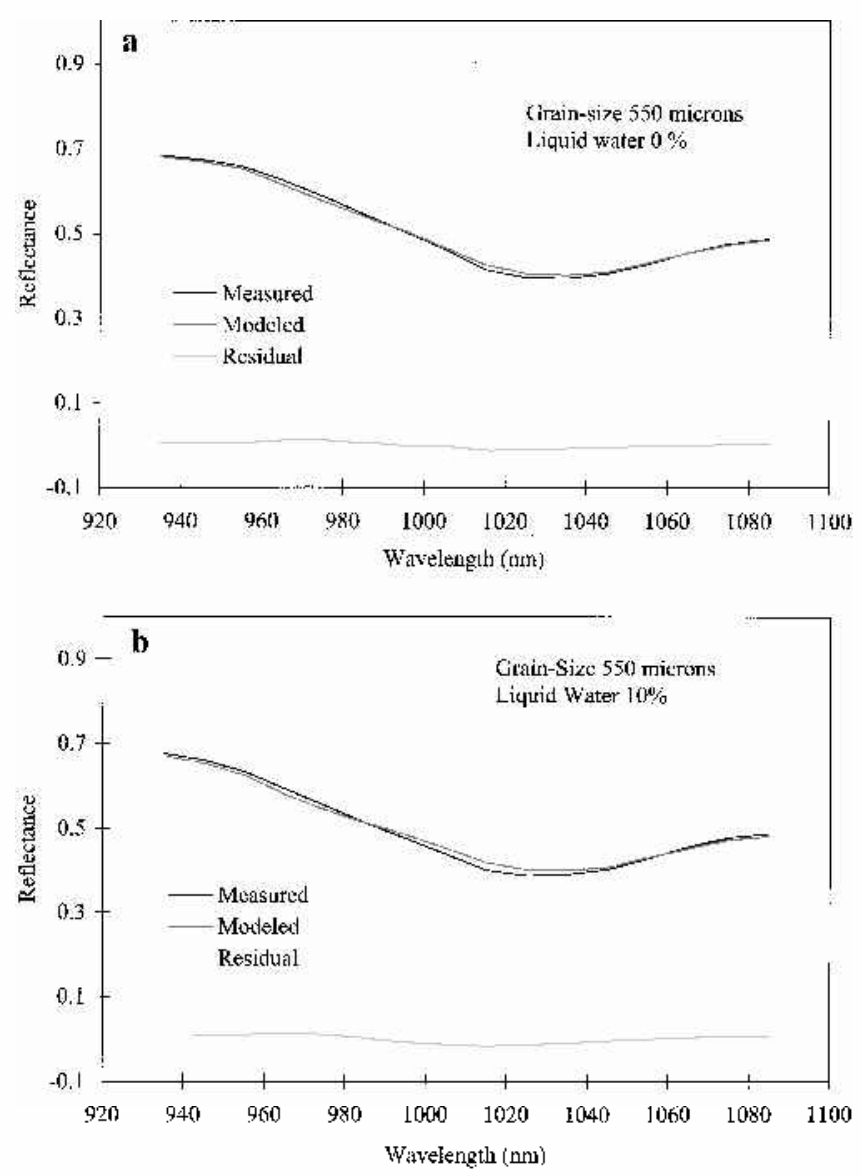

Fig. 5. Spectral fit of the coated-grain melting-snow model to the measured spectrum of (a) frozen snow and ( $b$ ) melting snow.

melt. Figure 4 shows the first spectrum of the frozen snow and 15 min spectrum after surface melting is well advanced. As predicted by the models, this measurement shows a shift of the $1030 \mathrm{~nm}$ absorption feature towards shorter wavelengths. Inspection of the measured frozen- and melting-snow spectra shows the shift in the $1030 \mathrm{~nm}$ feature to have a form more closely represented by the coated-sphere model.

To explore derivation of grain-size and liquid-water fraction from spectral snow-reflectance measurements, a spectral fitting algorithm was developed. A library of snow-reflectance spectra was calculated with the coatedsphere model for a grain-size range of $50-1500 \mu \mathrm{m}$ and a water-fraction range of $0-25 \%$. The library was then searched for the minimum residual difference between the frozen and melting measured spectra and every spectrum in the library. Figure $5 \mathrm{a}$ shows the best fit between the measured frozen-snow spectrum and the library. This fit was achieved with a value of $550 \mu \mathrm{m}$ grain-size and $0 \%$ liquid water. Figure $5 \mathrm{~b}$ shows the best fit for the meltingsnow spectrum. A value of $550 \mu \mathrm{m}$ grain-size and $10 \%$ liquid water was derived. This initial validation shows simultaneous derivation of grain-size and liquid-water content from measured snow-reflectance spectra using a spectral snow-reflectance model inversion.

\section{GONGLUSION}

Two models of the spectral reflectance of melting snow are described with initial validation results reported. The models are based on the complex refractive index of ice and liquid water. These fundamental optical properties were used with
Mie-theory codes to calculate the extinction, scattering and asymmetry parameters for two models of melting snow. In the first, the melting snow is modeled as interspersed spheres of ice and liquid water. In the second, the melting snow is modeled as coated ice spheres with liquid-water shells. These Mie-calculated optical properties were used to constrain DISORT to generate the spectral reflectance of snow with varying contents of liquid water. Both the coated- and interspersed-sphere models showed a shift of the absorption features centered at $1030 \mathrm{~nm}$ towards shorter wavelengths. This is consistent with the location of minima in the complex refractive index of ice vs liquid water.

Initial validation was performed by comparing the modeled snow spectra with measured spectra of frozen and melting snow. As with the model, the measured spectra showed a shift of the $1030 \mathrm{~nm}$ absorption features towards shorter wavelength. A spectral fitting algorithm was developed to invert for grain-size and liquid-water fraction using the coated-sphere model. This inversion algorithm produced grain-size and liquid-water fraction consistent with the expected snow properties.

This research shows that both the grain-size and melting status of snow can be derived with measurements from the solar-reflected portion of the spectrum. With the inclusion of atmospheric correction, terrain and bidirectional reflectance distribution function factors, this approach may be extended to airborne and spaceborne imaging spectrometer datasets at regional scales with global sampling. The resulting snow grainsize and melt-status maps would then contribute to energy balance and hydrological modeling and prediction efforts.

\section{ACKNOWLEDGEMENTS}

Part of this work was carried out at the Jet Propulsion Laboratory, California Institute of Technology, Pasadena, under contract with the National Aeronautics and Space Administration. Analyses and research were also carried out at the Institute for Computational Earth System Science at the University of California, Santa Barbara.

\section{REFERENGES}

Bohren, C. F. and B. R. Barkstrom. 1974. Theory of the optical properties of snow. 7. Geophys. Res., 79(30), 4527-4535.

Dozier, J. 1989. Spectral signature of alpine snow cover from the Landsat Thematic Mapper. Remote Sensing Environ., 28, 9-22, 159-163.

Kou, L. H., D. Labrie and P. Chýlek. 1993. Refractive indices of water and ice in the 0.65 to $2.5 \mu \mathrm{m}$ spectral range. Appl. Opt., 32(19), 3531-3540.

Nolin, A.W. and J. Dozier. 1993. Estimating snow grain size using AVIRIS data. Remote Sensing Environ., 44(2-3), 231-238.

Nolin, A. W. and J. Dozier. 2000. A hyperspectral method for remotely sensing the grain size of snow. Remote Sensing Environ., 74(2), 207-216.

Painter, T. H., D. A. Roberts, R. O. Green and J. Dozier. 1998. Improving mixture analysis estimates of snow-covered areas from AVIRIS data. Remote Sensing Environ., 65(3), 320-332.

Stamnes, K., S.-C. Tsay, W. Wiscombe and K. Jayaweera. 1988. Numerically stable algorithm for discrete-ordinate-method radiative transfer in multiple scattering and emitting layered media. Appl. Opt., 27(12), 2502-2509

Toon, O. B. and T. P. Ackerman. 1981. Algorithms for the calculation of scattering by stratified spheres. Appl. Opt., 20(20), 3657-3660

Warren, S. G. 1982. Optical properties of snow. Rev. Geophys. Space Phys., 20(1), 67-89.

Warren, S. G. and W. J.Wiscombe. 1980. A model for the spectral albedo of snow. II. Snow containing atmospheric aerosols. 7. Atmos. Sci., 37(12), 2734-2745.

Wiscombe, W. J. 1980. Improved Mie scattering algorithms. Appl. Opt., 19(9), 1505-1509.

Wiscombe, W. J. and S. G. Warren. 1980. A model for the spectral albedo of snow. I. Pure snow. 7. Atmos. Sci., 37(12), 2712-2733. 